\title{
THE $H$-DEVIATION OF A LIFT
}

\author{
FRANK WILLIAMS
}

(Communicated by Haynes R. Miller)

\begin{abstract}
Let $X$ be an $H$-space, $x \in X$ a primitive element, and $\alpha$ a stable primary operation that vanishes on $x$. Let $y$ represent $x$ in $H^{*}\left(P_{2} X\right) .\left(P_{2} X\right.$ is the projective plane of $X$.) Let $\tilde{D}$ be the $H$-deviation of the lift of $x$ to the two-stage Postnikov system with $k$-invariant $\alpha$. We obtain a formula that relates $\tilde{D}$ to the representation of $\alpha(y)$ as a product in $H^{*}\left(P_{2} X\right)$.
\end{abstract}

We consider the following data: $X$ a CW $H$-space, $g: K_{1} \rightarrow K_{0}$ a homomorphism of topological groups, and $(f, F): X \rightarrow \Omega K_{1}$ an $H$-map, such that $\Omega g \circ f$ is nullhomotopic. We thus have a diagram

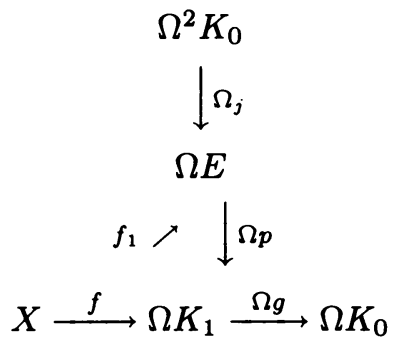

Here $E$ is the homotopy fiber of $g$ and the lift $f_{1}$ is given by

$$
f_{1}(x)=(f(x), l(x)) \in \Omega E \subset \Omega K_{1} \times P \Omega K_{0},
$$

where $l$ is a nullhomotopy $l: * \sim \Omega g \circ f$. Since $f$ is an $H$-map, the $H$-deviation $D f_{1}$ of $f_{1}$ factors as

$$
X \wedge X \stackrel{\tilde{D}}{\longrightarrow} \Omega^{2} K_{0} \stackrel{\Omega j}{\longrightarrow} \Omega E,
$$

where $\tilde{D}$ is a map we shall describe in detail below. In [3], Zabrodsky considered the above situation and proved (Proposition 3.2.2).

THEOREM $\mathrm{A}^{\prime}$. In the diagram $(*)$, suppose that

(a) $X$ is a loop space, $X=\Omega X^{\prime}$;

(b) $f$ is a loop map, $f=\Omega f^{\prime}$; and

(c) $g \circ f^{\prime}$ factors as

$$
X^{\prime} \stackrel{\bar{\Delta}}{\longrightarrow} X^{\prime} \wedge X^{\prime} \stackrel{\theta^{\prime}}{\rightarrow} K_{0}
$$

for some map $\theta^{\prime}$, where $\bar{\Delta}$ is the reduced diagonal.

Received by the editors October 16, 1987 and, in revised form February 20, 1988.

1980 Mathematics Subject Classification (1985 Revision). Primary 55P45, 55S40.

$K e y$ words and phrases. $H$-space, $H$-deviation, projective plane. 
If $l=\theta^{\prime} \circ \xi$, where $\xi$ is the standard nullhomotopy of $\Omega \bar{\Delta}$ then $\tilde{D}$ is adjoint to the composition $\theta$ given by

$$
\Sigma X \wedge \Sigma X \stackrel{\varepsilon \wedge \varepsilon}{\longrightarrow} X^{\prime} \wedge X^{\prime} \stackrel{\theta^{\prime}}{\longrightarrow} K_{0}
$$

where $\varepsilon$ is the evaluation map.)

In particular, there is the immediate corollary:

THEOREM $\mathrm{B}^{\prime}$. Under the hypotheses of Theorem $\mathrm{A}^{\prime}$, if $K_{0}$ is a generalized Eilenberg-Mac Lane space with field coefficients and

$$
\left[g \circ f^{\prime}\right]=\sum_{i}(-1)^{\operatorname{deg}\left(y_{i}^{\prime}\right)} y_{i}^{\prime} y_{i}^{\prime \prime} \in H^{*}\left(X^{\prime}\right)
$$

then by choosing $\theta^{\prime}$ such that

$$
\left[\theta^{\prime}\right]=\sum_{i} y^{\prime} \otimes y^{\prime \prime} \in H^{*}\left(X^{\prime} \wedge X^{\prime}\right)
$$

we get

$$
[\tilde{D}]=\sum_{i} \sigma^{*} y_{i}^{\prime} \otimes \sigma^{*} y_{i}^{\prime \prime} \in H^{*}(X \wedge X)
$$

These results have proved extremely useful in the study of $H$-spaces, but their utility is sometimes restricted due to the fact that hypotheses (a), (b), or (c) of Theorem $\mathrm{A}^{\prime}$ may not hold. In this paper we shall show that a formula for $\tilde{D}$ can be obtained in the absence of these hypotheses.

We establish some notation. Let

$$
\Sigma X \stackrel{h}{\rightarrow} P_{2} X \stackrel{\hat{\Delta}}{\longrightarrow} C_{h} \sim \Sigma^{2}(X \wedge X)
$$

define $C_{h}$ as the mapping cone of the inclusion of the suspension in the projective plane. Let $F: P_{2} X \rightarrow K_{1}$ be induced by the $H$-structure of $f$. (We shall justify this notation below.)

THEOREM A. The map $g \circ F$ factors as $\theta \circ \hat{\Delta}$, where $\theta: \Sigma^{2}(X \wedge X) \rightarrow K_{0}$ is adjoint to $\tilde{D}$.

Theorem A has the immediate corollary (using pp. 495-496 of [1]):

THEOREM B. Let $K_{0}$ be a generalized Eilenberg-Mac Lane space with field coefficients

(a) If $[g \circ F]=\sum_{i}(-1)^{\operatorname{deg}\left(z_{i}^{\prime}\right)} z_{i}^{\prime} z_{i}^{\prime \prime} \in H^{*}\left(P_{2} X\right)$, then

$$
[\tilde{D}]=\sum_{i} h^{*} z_{i}^{\prime} \otimes h^{*} z_{i}^{\prime \prime} \in H^{*}(X \wedge X)
$$

modulo $\operatorname{Im}\left(\bar{\mu}^{*}\right)$.

(b) Conversely, if

$$
[\tilde{D}]=\sum_{i} x_{i}^{\prime} \otimes x_{i}^{\prime \prime}
$$

then

$$
\left[g \circ F \left[=\hat{\Delta}^{*}\left(\sum_{i} \otimes x_{i}^{\prime} \otimes x_{i}^{\prime \prime}\right) .\right.\right.
$$


Hence, if the $x_{i}^{\prime}$ and $x_{i}^{\prime \prime}$ are primitive and $x_{i}^{\prime}=h^{*}\left(z_{i}^{\prime}\right), x_{i}^{\prime \prime}=h^{*}\left(z_{i}^{\prime \prime}\right)$, then

$$
[g \circ F]=\sum_{i}(-1)^{\operatorname{deg}\left(z_{i}^{\prime}\right)} z_{i}^{\prime} z_{i}^{\prime \prime}
$$

We remark that the indeterminacy of $[\tilde{D}]$ resulting from different choices of the nullhomotopy $l$ consists of exactly $\operatorname{Im}\left(\bar{\mu}^{*}\right)$. Hence there exists a lift $f_{1}$ with precisely the $H$-deviation given by Theorem $\mathrm{B}$.

Before turning to the proof of Theorem A, let us make explicit the way in which our theorem extends that of Zabrodsky. Under the hypotheses of Theorem $\mathrm{A}^{\prime}$, the evaluation map $\varepsilon$ factors as

$$
\Sigma X \stackrel{h}{\rightarrow} P_{2} X \stackrel{k}{\rightarrow} X^{\prime}
$$

as described on pp. 18-19 of [2]. As in Theorem B', write

$$
\left[g \circ f^{\prime}\right]=\sum_{i}(-1)^{\operatorname{deg}\left(y_{i}\right)} y_{i}^{\prime} y_{i}^{\prime \prime} \in H^{*}\left(X^{\prime}\right) \text {. }
$$

To apply Theorem B, we set $F=f^{\prime} \circ k$, so that

$$
[g \circ F]=\sum_{i}(-1)^{\operatorname{deg}\left(z_{i}\right)} z_{i}^{\prime} z_{i}^{\prime \prime} \in H^{*}\left(P_{2} X\right),
$$

where $z_{i}^{\prime}=k^{*}\left(y_{i}^{\prime}\right)$ and $z_{i}^{\prime \prime}=k^{*}\left(y_{i}^{\prime \prime}\right)$. By Theorem B,

$$
[\tilde{D}]=\sum_{i} h^{*} z_{i}^{\prime} \otimes h^{*} z_{i}^{\prime \prime}=\sum_{i} \sigma^{*} y_{i}^{\prime} \otimes \sigma^{*} y_{i}^{\prime \prime},
$$

modulo $\operatorname{Im}(\bar{\mu})$. Thus, up to the indeterminary of $f_{1}$, Theorem $\mathrm{B}^{\prime}$ follows from Theorem B.

We wish to thank James P. Lin for suggesting the problem considered in this note. We also wish to thank the University of California-San Diego for it's hospitality during the time this work was done. The rest of the paper is devoted to the proof of Theorem A.

Let $\Delta^{n}$ denote the standard $n$-simplex. We may represent the $H$-structure of the map $f: X \rightarrow \Omega K_{1}$ as

$$
F: \Delta^{2} \times X^{2} \rightarrow K_{1}
$$

such that

$$
\begin{aligned}
& F\left(t_{0}, t_{1}, 0, x_{1}, x_{2}\right)=f\left(x_{1}\right)\left[t_{0}\right], \\
& F\left(0, t_{1}, t_{2}, x_{1}, x_{2}\right)=f\left(x_{2}\right)\left[t_{1}\right], \\
& F\left(t_{0}, 0, t_{2}, x_{1}, x_{2}\right)=f\left(x_{1} x_{2}\right)\left[t_{0}\right], \\
& F\left(t_{0}, t_{1}, t_{2}, *, x_{2}\right)=f\left(x_{2}\right)\left[t_{0}+t_{1}\right],
\end{aligned}
$$

and

$$
F\left(t_{0}, t_{1}, t_{2}, x_{1}, *\right)=f\left(x_{1}\right)\left[t_{0}\right] .
$$

Checking the construction of $P_{2} X$ as a quotient space of $\Delta^{2} \times X^{2}[2$, p. 8] we see that $F$ respects precisely those identifications and hence may be regarded as a $\operatorname{map} F: P_{2} X \rightarrow K_{1}$. 
The map $\tilde{D}: X \wedge X \rightarrow \Omega^{2} K_{0}$ is given by the formula (cf. [3, p. 73]):

$$
\tilde{D}\left(x_{1}, x_{2}\right)=l\left(x_{1} x_{2}\right)+P \Omega g \circ F\left(x_{1}, x_{2}\right)-l\left(x_{1}\right) l\left(x_{2}\right),
$$

in which juxtaposition denotes multiplication in $X$ and in $\Omega K_{0}$ and addition denotes the concatenation of paths. The adjoint of $\tilde{D}$ can be expressed as a map

$$
\theta=\dot{\Delta}^{3} \times X^{2} \rightarrow K_{0}
$$

given by the picture:

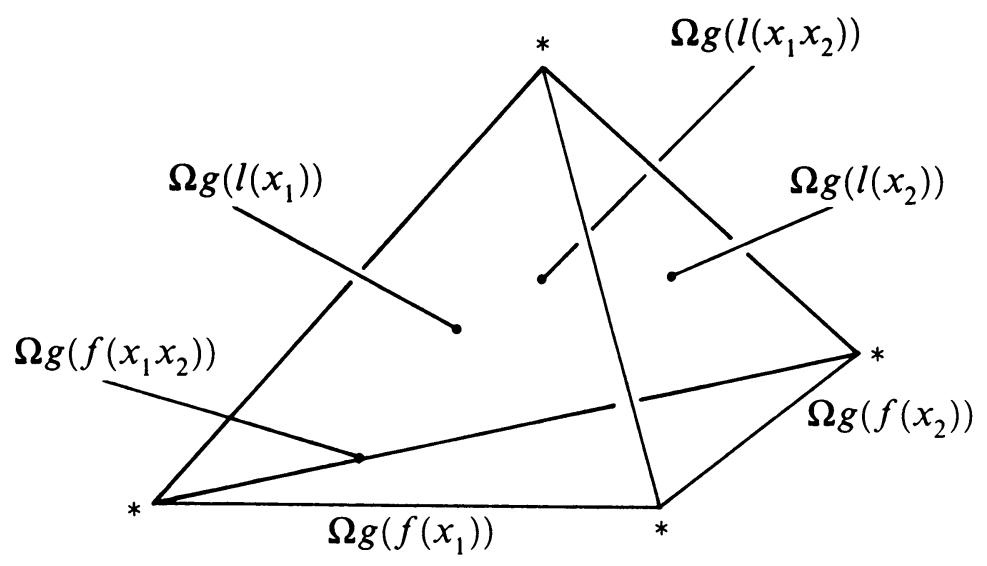

FIGURE 1

Consider the diagram

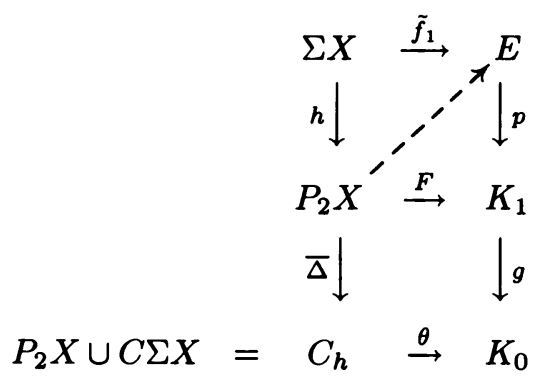

in which $\tilde{f}_{1}$ is the adjoint of $f_{1}$. The space $C_{h}$ may be represented as a quotient of $\dot{\Delta}^{3} \times X^{2}$ by coordinates

$$
P_{2} X=\left\{\left(t_{0}, t_{1}, t_{2}, 0, x_{1}, x_{2}\right)\right\}
$$

subject to the appropriate identifications, and

$$
C \Sigma X=\left\{\left(t_{0}, t_{1}, t_{2}, t_{3}, x_{1}, x_{2}\right) \mid t_{i}=0, i=0,1,2\right\}
$$

with $t_{3}$ as the cone parameter. Clearly $\theta$ makes the lower square commute. (In fact, from standard obstructioin theory $\theta$ is exactly the obstruction to the existence of a diagonal arrow that makes both triangles of the upper square commute.) Theorem A follows. 


\section{REFERENCES}

1. W. Browder and E. Thomas, On the projective plane of an $H$-space, Illinois J. Math. 7 (1963), 492-502.

2. J. D. Stasheff, $H$-spaces from a homotopy point of view, Lecture Notes in Math., vol. 161, Springer-Verlag, Berlin, Heidelberg and New York, 1970.

3. A. Zabrodsky, Hopf spaces, North-Holland, Math. Study \#22, North-Holland, 1976.

Department of Mathematics, New Mexico State University, las Cruces, NEW MEXICO 88003 